\title{
ФОРМИРОВАНИЕ ТРЕБОВАНИЙ К АВТОМАТИЗИРОВАННОЙ СИСТЕМЕ КОНТРОЛЯ СОСТОЯНИЯ СОВРЕМЕННЫХ КОСМИЧЕСКИХ АППАРАТОВ
}

\section{FORMATION OF REQUIREMENTS FOR AN AUTOMATED SYSTEM FOR MONITORING THE STATE OF MODERN SPACECRAFT}

\section{S. Solovyov}

Summary. This article discusses the development of an automated system for monitoring the state of spacecraft. The necessity of development of automated state control tools is identified and justified. Based on the research, the author suggests an approach to developing a system that allows automating routine tasks of a control group specialist in terms of analyzing telemetry information, visually displaying the results of automated state analysis, and implementing interactive information support for a specialist in the operational shift of the spacecraft flight control. The requirements for the most popular automation tasks are formalized in terms of the needs of specialists in operational flight control of the SPACECRAFT. The focus is on solving the problem of automating the process of analyzing telemetry information from the spacecraft.

Keywords: automated control system, spacecraft, monitoring, state analysis, flight control, telemetry information.

\section{Ввемение}

$\mathbf{T}$ екущими направлениями развития космонавтики является совершенствование космических аппаратов (КА), в том числе и пилотируемых кораблей, как технического устройства, расширение количества выполняемых КА функциональных задач и как следствие увеличение полетных операций в процессе выполнения орбитального полета. Что в свою очередь усложняет задачу управления полетом, особенно с учетом возрастания длительности функционирования КА.

Контроль является необходимой задачей решаемой при управлении полетом КА, которая играет роль обратной связи, с помощью которой оценивается состояние КА и выполняемая программа полета. Оценке подлежат все основные процессы, происходящие на борту КА. Оценка состояния и работы составных частей КА является основной функцией для обеспечения работоспособности КА, рационального использования его ресурсов, требуемого характера управляемого движения, выпол-

\author{
Соловьев Сергей Владимирович \\ К.т.н., Ведущий конструктор, ПАО РКК «Энергия» \\ С. П. Королёва \\ sergey.soloviev@scsc.ru
}

Аннотация. В данной статье рассмотрен вопрос разработки автоматизированной системы контроля состояния космических аппаратов. Выявлена и обоснована необходимость развития автоматизированных средств контроля состояния. На основе проведенного исследования автором предлагается подход к разработке системы, которая позволяет автоматизировать рутинные задачи специалиста группы управления в части анализа телеметрической информации, наглядно отображать результаты автоматизированного анализа состояния и реализует интерактивную информационную поддержку специалиста оперативной смены управления полетом космического аппарата. Формализованы требования для наиболее востребованных задач автоматизации с точки зрения потребностей специалистов оперативного управления полетом КА. Делается акцент на решение задачи по автоматизации процесса анализа телеметрической информации с борта космического аппарата.

Ключевые слова: автоматизированная система контроля, космический аппарат, контроль, анализ состояния, управление полетом, телеметрическая информация.

нения им полётных операций, научно-исследовательской программы и плана полета в целом [1, 2, 3, 4].

Технология контроля состояния КА, является достаточно отработанной, и включает устоявшуюся последовательность действий, выполняемую как аппаратно-программными средствами, так и операторами службы управления.

На процесс и технологию контроля состояния КА существенное влияние оказывают параметры орбитального движения КА, конструктивные особенности КА и наземного контура управления (НКУ). Это выражается в наличии ограничений:

- по длительности сеанса связи;

- пропускной способности радиолинии передачи телеметрической информации (ТМИ) от КА на НKУ;

- возможностей датчиковой аппаратуры, бортовых средств измерений и вычислительных средств KA. 
Перечисленный ограничения определяются и учитываются на этапе создания КА. Принимаются технические решения, оптимизирующие влияния этих факторов на процесс управления полетом КА [5]. Тем не менее, общая тенденция увеличения функциональных возможностей КА, длительности орбитального полета и производительности бортовых вычислительных средств КА приводят к увеличению количества разнородной информации, поступающей от КА и контролируемой персоналом управления.

Значительный опыт управления полетом КА различного типа и назначения, накопленный в настоящее время, позволяет четко сформулировать основные недостатки средств и технологий контроля, которыми в настоящее время являются:

- наличие операций «ручного» контроля;

- ограниченная автоматизация анализа для контроля выполнения полетных операций;

- отсутствие автоматизированного прогнозирования технического состояния КА;

- ограничение по отображению ТМИ на рабочем месте оператора;

- ограниченная информационная поддержка оператора.

В статье представлен подход к разработке автоматизированной системы контроля состояния КА создаваемой с целью минимизации человеческого фактора в процессе управления полетом КА, повышению информационной доступности и снижению нагрузки специалиста группы анализа на выполнение рутинных операций. Отличительной особенностью предлагаемого подхода является реализация концепции максимальной автоматизации процессов анализа ТМИ КА и в результате представления специалисту управления только результатов анализа. Значительно расширяются возможности системы по быстрому представлению разнообразных данных из эксплуатационной и конструкторской документации, с реализацией трехмерного объемного отображения конструкции и компоновки КА и его составных частей, приборов и агрегатов.

\section{ПреАлагаемый по ххо \\ к разработке автоматизированной системе кOHтро^Я}

Автоматизированная система контроля (АСК) КА предназначена для автоматизированного решения задач анализа и информационной поддержки специалистов оперативной смены при управлении полетом современных и длительно эксплуатируемых КА, в том числе в составе их орбитальной группировки [6].

В соответствии с назначением, АСК решает следующие задачи:
- прием полного потока ТМИ КА;

- устранение сбойности, привязка к шкале времени, обработка ТМИ до уровня физических значений параметров;

- отображение ТМИ на автоматизированных рабочих местах специалистов оперативной смены управления полетом КА;

- автоматизированный и интеллектуализированный анализ ТМИ КА;

- автоматизированное формирование рекомендаций при выявлении нештатных ситуаций (НШС) на борту КА;

- прогнозирование состояния составных частей КА;

- учет расходования и запасов ресурсов КА;

- автоматизированное формирование отчетов по результатам обработки ТМИ в интересах оперативной смены управления;

- информационной поддержки специалистов оперативной смены, как единой интерактивной электронной системы документации;

- хранение полученных результатов.

Важными предпосылками для автоматизации процесса контроля при управлении полётом КА являются:

- необходимость устранения множества рутинных постоянно повторяющихся операций, выполнение которых специалистами управления поелтом КА может быть заменено работой автоматизированной системы;

- обработка большого количества данных и мониторинг огромного, для человеческих возможностей, числа параметров состояния для получения достаточного объёма информации для принятия решений при управлении полётом;

- длительность полётов и накопление значительного количества информации в процессе эксплуатации (например, МКС функционирует уже на протяжении 20 лет);

- большая трудоемкость существующих технологий и ее возрастание при конструктивном и количественном росте КА, особенно для многоспутниковых группировок или орбитальных комплексов сложной архитектуры;

- высокая производительность вычислительных средств НКУ;

- значительных прогресс в информационных технологиях и интеллектуализированной обработки данных.

В статье рассматривается формализация требований к наиболее актуальным, технически сложным и востребованным задачам автоматизации, с точки рения решения практических задач управления полетом КА, а именно:

- отображение ТМИ на автоматизированных рабочих местах специалистов оперативной смены управления полетом КА; 
- автоматизированный и интеллектуализированный анализ ТМИ;

- информационная поддержка специалистов оперативной смены управления полетом КА.

\section{Формализашия требований к отображению ТМИ}

Типичная система отображения ТМИ для информативного представления состояния КА, реализована с использований специальных форматов отображения. В форматах содержится обозначение телеметрического параметра (ТМП), его текущее значение и время формирования этого значения. Для значительного числа ТМП такое представление является достаточно стандартным для большинства разнотипных КА. Задача представления информации является очень востребованной на практике. Это обусловлено физиологическими возможностями человека, что ограничивает длительность концентрации его внимания и сокращает объем одновременно осмысленно контролируемых данных. Это приводит к необходимости задействования сразу нескольких квалифицированных специалистов. Исходя из перечисленных обстоятельств для отображения обработанной ТМИ КА используется иерархическая структурированная система форматов отображения на экранах автоматизированных рабочих местах операторов службы управления. Структура форматов рассчитывается таким образом, чтобы обеспечить отображение всего объема обработанной ТМИ возможно на экране одного рабочего места.

Базовыми требованиями к отображению обработанной ТМИ на экранах рабочих мест операторов службы управления являются:

- структурированность отображаемой информации, как правило по принадлежности параметров к отдельным составным частям КА;

- количество информации на формате отображения для разумного восприятия оператором;

- единая форма представления последовательности - параметр или индекс параметра, значение, время формирования значения параметра с привязкой к бортовой шкале времен.)

- цветоотображение информации для акцентированного восприятия:

- фон «экологичный», светлый;

- данные темнее;

- цветовая индикация значения (зеленый, желтый, красный по принципу светофора);

- последовательность представления информации от общего к частному.

Структурирование отображаемой информации по иерархическому принципу позволяет разнести всю совокупность форматов отображения обычно на три уровня:

- форматы обобщенного контроля состояния КА в целом и/или выполнения программы полета;

- форматы обобщенного контроля по каждой составной части КА;

- форматы отображения значений ТМП всего объема ТМИ КА по соответствующим составным частям КА.

Важным свойством средств отображения является универсальность для необходимо реализовать:

- иерархическое отображение формуляров по принципу связности от одного (общего) к другому (частный);

- ретрансляция (передача) обобщенной информации по результатам работы алгоритма анализа от частного формуляра к общему;

- формирования отдельного перечня исполняемых алгоритмов с однозначным параметрическим описанием;

- документирование всех действий при проведении работы алгоритмов.

Отображаемая на экранах рабочих мест специалистов управления тем или иным образом ТМИ в конечном итоге подвергается анализу непосредственно специалистами. Поскольку анализу подвергается большой объем разнородной информации, большое значение приобретает квалификация и опыт специалиста управления. В связи с этим существует необходимость в разработке автоматизированных методов, интуитивно наглядном для специалистов оперативной смены управления полетом, позволяющих исследовать информацию для достижения тех или иных результатов в зависимости от целей или фазы космического полета КА.

\section{Формализашия требований к интем мектуализированному ана^из ТМИ}

Сущность интеллектуализации анализа ТМИ КА, является получение новых знаний о процессах происходящих при функционировании аппаратуры, составных частей КА и КА в целом. Детальное понимание состояния и изменения процессов на борту КА, а также определения параметров взаимовлияния составных частей КА друг на друга позволяет глубоко познать свойства и процессы в КА и его составных частей в условиях реальной эксплуатации со всеми внешними возмущениями. Работа интеллектуализированной системы в режиме реального времени увеличить скорость реакции на появление аномалий и их развитие во времени с четкой фиксации направления развития отклонения в данных ТМИ. Поэтому в части интеллектуального 
анализа ТМИ требования формализованы следующем образом.

Интеллектуализированный анализ предназначен для оценки текущего состояния КА, определения отклонений или аномалий от номинального или планируемого состояния в работе составных частей (СЧ) КА и выявление НШС, автономно без оперативных действий специалистов оперативной смены управления полетом КА.

Интеллектуализированный анализ ТМИ должен включать в себя:

- комплексную оценку состояния КА с формированием сообщения «норма/ненорма»;

- оценку состояния и функционирования каждой СЧ КА с формированием сообщения «норма/ненорма СЧ КА»;

- автоматически обнаружение НШС в отдельных СЧ KA;

- локализация (определения места, прибора или агрегата) возникновения отклонения или НШС;

- определение причины обнаруженного отклонения или НШС;

- формирование перечня НШС, которые могут быть вызваны обнаруженной аномалией;

- выявление тенденций изменения значения ТМП, с формированием «располагаемого времени» до достижения предельных значений ТМП;

- определение готовности КА к выполнению программы полета;

- формирование запроса специалистов оперативной смены управления полетом КА в случае обнаружения НШС:

В структуре задач контроля при управлении полетом КА можно сформировать следующие задачи интеллектуального анализа:

- автоматизация процедур анализа ТМИ;

- извлечение неочевидных знаний о функционировании и взаимовлиянии составных частей КА при изменяющихся внешних воздействующих фактоpax;

- выявление, локализация и определение причин аномалий, нечетких состояний или нерасмотренных НШС;

- прогнозирование состояния КА и его отдельных характеристик на определенную перспективу;

- идентификация и построение прогнозирующих идентификационных моделей.

Сущностью решения задачи автоматизации процессов контроля является:

- повышение оперативности, качества и надежности управления в части решения задач контроля состояния и функционирования КА;
- повышение производительности труда специалиста управления полетом, т.е. решение необходимого объёма задач меньшей численностью единовременно привлекаемых специалистов управления;

- снижение нагрузки на специалиста управления полетом и её перераспределение с рутинных постоянно повторяющихся операций на задачи более высокого интеллектуального уровня.

Достигается это за счет устранения «человеческого фактора» и его ограниченных возможностей при переработке информации и что более существенно, в большой зависимости от квалификации специалиста на скорость решения задач анализа особенно при оперативном управлении полетом КА.

Задачи повышение оперативности решения задач анализа достигаются средствами автоматизации путем расширения возможностей персонала управления в процессе эксплуатации КА с помощью технических и программных средств вычислительной техники.

Различные методы автоматизации позволяют формировать, так называемых, обобщенных параметров. Данные параметры формируют интегральную оценку, которая характеризует состояние отдельной СЧ КА. Общий принцип подобных методов состоит в определении значений всех ТМП внутри допустимого диапазона и формировании сообщения «норма», а при выходе текущих значений какого-либо параметра за допустимый диапазон, сообщения «не норма». Для исключения сбоев первичные параметры от датчиков разбивают на группы и анализируют по мажоритарной схеме. Это позволяет исключить ошибки, связанные с выходом из строя отдельных датчиков, или сбои при передаче данных с борта КА на наземные средства обработки.

Иерархическое объединение обобщенных параметров по СЧ КА по принципу «от простого к сложному» и дополнительные алгоритмы обработки позволяют в конечном итоге образовать обобщенный параметр на уровне КА в целом. Это весьма эффективное решение особенно при длительных рабочих режимах КА, характерных для КА связи, метеорологии и навигации.

Автоматизация рутинных операций процесса контроля наземными техническими средствами управления, позволила существенно сократить персонал ЦУП управления КА связи, до одного-двух операторов на 5-6 КА, без потери качества выполнения контрольных функций и надежности управления в целом.

Наличие специализированных алгоритмов анализа позволит оперативно выявлять сбои и кратковремен- 
ные отклонения в функционировании КА, оптимизировать формирование и выдачу управляющих воздействий, тем самым минимизируя изменения программы полета. Большое значение будет иметь возможность использовать в них информацию, получаемую при полете однотипных КА, и опыт их эксплуатации.

\section{Формализация требований к информашионной по Аержке}

Одним из существенных факторов, направленных на повышение эффективности контроля состояния современных КА, является информационная поддержка специалистов оперативной смены управления полетом КА. Исходя из анализа их действий и решаемых задач, представляются весьма востребованными и актуальными следующие виды информационной поддержки:

- информационно-поисковые системы;

- трехмерные модели конструкции КА;

- вспомогательные модели (баллистические, динамические и т.п).

Информационно-поисковые системы призваны упростить работу специалистов оперативной смены с эксплуатационной документацией [18-20]. Объем эксплуатационной документации по управлению современным КА составляет от 50 до 100 различных источников (инструкции, технические описания, журналы смен и т.п.). Все виды документации (проектная, конструкторская, эксплуатационная и др.) сейчас разрабатываются, хранятся и распространяются в электронном виде. Вполне закономерным видится предложение использовать данную документацию также в безбумажном виде. Однако самое существенное в технологии её использования - это быстрый поиск необходимой информации с нечеткими признаками в большом объеме электронных документов. Поэтому с рабочего места специалистов оперативной смены целесообразно иметь доступ к специальному поисковому программному обеспечению, позволяющему по различным критериям находить необходимые сведения в эксплуатационной и в других видах документации. При этом должны быть реализованы следующие способы поиска информации:

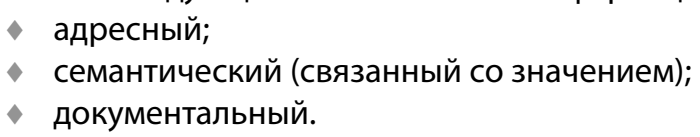

Отдельно следует отметить необходимость поиска информации по телеметрическим параметрам не только в инструкциях по эксплуатации, но и технических описаниях и по возможности в конструкторских документах. Поэтому в дальнейшем будем под понятием поиск информации понимать - процесс, в ходе которого в той или иной последовательности производится соотнесение отыскиваемого с каждым документом, хранящимся в системе. Информационно-поисковые системы достигли значительного совершенства и позволяют, при соответствующей настройке, весьма эффективно осуществлять поиск в массивах машиночитаемых данных, содержащих заданные слова.

Для специалистов оперативной смены характерна устойчивость тематического профиля запросов, то есть запросы являются «информационно-ориентированными», и соответственно есть возможность организовать информационное пространство проблемы. Это означает, что пользователь создаёт по существу новый, «самостоятельный» проблемно-ориентированный, индивидуально обновляемый и пополняемый информационный подресурс, включающий помимо подборок документов также и метаинформацию, например, словари специальной терминологии, классификаторы предметных областей, описания ресурсов и т.д.

Существенными факторами для понимания методов автоматизации поиска являются два следующих фактоpa:

- сравниваются не сами объекты, а описания - так называемые «поисковые образы»;

- сам процесс является сложным (составным и не одноактным) и обычно реализуется последовательностью операций.

Данные в информационно-поисковую систему должны вводиться на основе специально разрабатываемых форматов ввода.

Термины и телеметрические параметры, применяемые для управления полетом КА, как правило, являются составными, т.е. содержат несколько слов, поэтому организация поиска должна происходить с усечением справа. Это общепризнанная форма организации в поисковых машинах типа «Google» и т.п. применяемых в сети Интернет. Например, при вводе в строку поиска «температура на торцевой панели 21», после набора слова «температура» информационно-поисковая машина отображает ссылки на параметры содержащие это слова. При добавлении следующего слова, происходит уточнение и остаются ссылки только на параметры температуры торцевой панели и т.д. В каждом случае оператор должен представлять, что именно он хочет найти, так как в предложенном ему варианте будет найдено гораздо большее количество документов, чем при задании данного слова полностью (без усечения). В подобном случае возможно в полученном массиве информации провести уточняющий поиск, например, структурированный в соответствии со схемой деления КА и в результате получить более адекватные и точные данные.

Исходя из изложенного выше, можно формализуются требования к единой интерактивной электронной 
системе представления документации (ЕСД), которая должна обеспечивать:

- оперативный поиск информации во всём массиве документации;

- автоматический выбор необходимой документации, используемой в текущий момент времени реализации плана полета;

- структурирование документации на уровни и подуровни (оперативная документация, справочная информация, организационная документация, процедуры при НШС, графическая информация и др.);

- предоставление более информативной графической информации (3D анимация, видео информация);

- наличие единых актуальных версий документации;

- использование современных методов и алгоритмов компьютерной обработки информации, представленной в документации, с целью выработки своевременных решений и подсказок для специалистов оперативной смены;

- увязывание документации с текущими показаниями ТМИ при возникновении НШС;

- возможности на программном уровне взаимодействовать с другими информационными системами, например, автоматизированной системой планирования и др.;
- доступность всей документации на всех рабочих местах специалистов оперативной смены.

\section{Выво $\triangle \mathrm{b}$}

Рассмотренные в данной статье задачи автоматизации процессов контроля при управлении полетом современных КА не являются исчерпывающими. В зависимости от целевого назначение КА или орбитальной группировки КА могут изменяться не только задачи и их совокупный набор, но также появляться специфические и самостоятельные направления. Учитывая сложившуюся практику управления полетом в российской космонавтике, в целом сформирован подход к разработке автоматизированной системы контроля состояния КА. Автоматизация и интеллектуализация решения этих задач позволяет повысить качественный уровень функционирования специалистов оперативной смены. В том числе это достигается за счет извлечения новых знаний о свойствах, процессах и состоянии КА, что тем самым позволяет глубже понять и дать возможность повлиять на происходящее. за счет выявления аномальных ситуаций потенциально способных перейти в НШС. Оперативное обеспечение информацией специалистов оперативной смены по широкому кругу решаемых задач благотворно влияет на надежность и оперативность процесса управления в целом.

\section{ЛИТЕРАТУРА}

1. В.А. Соловьёв, Л. Н. Лысенко, В. Е. Любинский. Управление космическими полетами: учеб. пособие: в 2 ч. // Ч. 2 М.: МГТУ им. Н. Э. Баумана, 2010. - 426 с.

2. Кравец В. Г., Любинский В. Е. Основы управления космическими полётами. М.: Машиностроение, 1983. 256 с.

3. Управление полетом орбитальной станции «Мир». Концепция автоматизированного планирования и управления / А. П. Ушаков, А. Н. Брега, А. А. Коваленко, С. Г. Чернобровкин. 115-9/87.— Калининград: РКК «Энергия» им. С. П. Королева, 1995.— 243 с.

4. Антонов Г., Курочкин В. Некоторые теоретические аспекты аварийности с точки зрения практики._- Морской сборник, 1994, № 6.

5. Наземный комплекс управления дальними космическими аппаратами. Перспективы развития/Под ред. Ю. М. Урличича. М.: Радиотехника, 2012.216 с.

6. Соловьев С. В., Мишурова Н. В. Анализ текущего состояния процесса контроля при управлении полетом космических аппаратов. Инженерный журнал: наука и инновации, 2016, вып. 3. URL: http://engjournal.ru/catalog/arse/adb/1474.html D0l 10.18698/2308-6033-2016-03-1474

7. Бочкарев В. В., Крыжановский Г. А., Сухих Н. Н. Автоматизированное управление движением авиационного транспорта // М.: Транспорт, 1999.

8. Медведев А. В., Сухих Н. Н., Федоров М.С. Структуры и алгоритмы управления сложных авиационных систем//Структуры сложных систем и алгоритмы управления/Под ред. Ю. А. Борцова, В. Б. Яковлева (Вопросы теории систем автоматического управления; Вып.8). -Л.: Изд-во Ленингр. ун-та. 1990.

9. Бровкин А. Г., Бурдыгов Б. Г., Гордийко С. В. и др. Под редакцией А. С. Сырова, Бортовые системы управления космическими аппаратами: Учебное пособие - М.: Изд-во МАИ-ПРИНТ, 2010. - 304 с.

10. Шкляр В. Н. Надежность систем управления:Учебное пособие. — Томск: Изд-во Томского политехнического университета, 2009. - 126 с.

11. Лукин Ф. А., Шахматов А. В., Мушовец К. В. и др. Механизм управляемой телеметрии космического аппарата // Вестник СибГАУ. 2012 . № 5(45). С. 140-144.

12. Современная телеметрия в теории и на практике /А.В. Назаров, Г. И. Козырев, И. В. Шитов и др:- СПб.; Наука и техника, 2007.- 672с.

13. Милицин А. В., Самсонов В. К., Ходак В. А. Отображение информации в центре управления космическими полетами — М.: Радио и связь, 1982.-190с.

14. Князев А. В. Теоретико-графовое моделирование информационных процессах в АСУ испытаниях //вестник Московского авиационного института. 2002. Т 9. № 2. C. 38-43.

15. Гасов В. М., Коротаев А. И., Сенькин С. И. Отображение информации. - М.: Высшая школа, 1990. - 111 с. 